\title{
Spontaneous and Fungicide-Induced Genomic Variation in Sclerotinia sclerotiorum
}

\author{
Nikita Gambhir, Zhian N. Kamvar, Rebecca Higgins, B. Sajeewa Amaradasa, and Sydney E. Everhart ${ }^{\dagger}$
}

Department of Plant Pathology, University of Nebraska, Lincoln, NE 68583

Accepted for publication 13 November 2020.

\begin{abstract}
Stress from exposure to sublethal fungicide doses may cause genomic instability in fungal plant pathogens, which may accelerate the emergence of fungicide resistance or other adaptive traits. In a previous study, five strains of Sclerotinia sclerotiorum were exposed to sublethal doses of four fungicides with different modes of action, and genotyping showed that such exposure induced mutations. The goal of the present study was to characterize genome-wide mutations in response to sublethal fungicide stress in S. sclerotiorum and study the effect of genomic background on the mutational repertoire. The objectives were to determine the effect of sublethal dose exposure and genomic background on mutation frequency/ type, distribution of mutations, and fitness costs. Fifty-five $S$. sclerotiorum genomes were sequenced and aligned to the reference genome. Variants were called and quality filtered to obtain high confidence calls for single nucleotide polymorphisms (SNPs), insertions/deletions (INDELs), copy number variants, and transposable element (TE) insertions. Results
\end{abstract}

ABSTRACT suggest that sublethal fungicide exposure significantly increased the frequency of INDELs in two strains from one genomic background $(P$ value $\leq 0.05$ ), while TE insertions were generally repressed for all genomic backgrounds and under all fungicide exposures. The frequency and/or distribution of SNPs, INDELs, and TE insertions varied with genomic background. A propensity for large duplications on chromosome 7 and aneuploidy of this chromosome were observed in the $S$. sclerotiorum genome. Mutation accumulation did not significantly affect the overall in planta strain aggressiveness $(P$ value $>0.05)$. Understanding factors that affect pathogen mutation rates can inform disease management strategies that delay resistance evolution.

Keywords: aneuploidy, experimental evolution, fungicide stress, genomic background, mutation accumulation, mutation rate, resistance, resistance emergence, sublethal dose, transposable elements, white mold
Fungicides play a key role in crop protection. Without fungicides, yield of certain crops (e.g., grapes, papaya, and pear) would be reduced by $\geq 95 \%$ (Gianessi and Reigner 2005). Most fungicides used today have a single-site mode of action, meaning that they inhibit a particular biochemical pathway by binding to a target site in the fungal cell. This specificity makes them effective but also increases the potential of pathogens evolving resistance to these fungicides. A single mutation in the DNA sequence of the target site may change its binding affinity and render the fungicide ineffective. Fungicide resistance has already been reported for 203 plant pathogenic fungi (Fungicide Resistance Action Committee 2012). It takes approximately $\$ 286$ million (inflation-adjusted to $\$ 315$ million) and 11 years to develop and market a new fungicide (McDougall 2016), but resistance was reported as early as 2 years after the launch of some fungicides (Brent and Hollomon 2007). In order to delay resistance evolution and prolong the life of currently used fungicides, it is important to understand the role of different

†Corresponding author: S. Everhart; everhart@unl.edu

Current address for Z. N. Kamvar: The Carpentries, Oakland, CA 94607.

Current address for B. S. Amaradasa: The Institute for Advanced Learning and Research, Danville, VA 24540.

Funding: Support was provided by the University of Nebraska Agriculture Research Division to S. Everhart, USDA-ARS National Sclerotinia Initiative grant number 58-5442-2-209, and North Central Soybean Research Program grant number 639K623. This work was partially completed utilizing the Holland Computing Center of the University of Nebraska, which receives support from the Nebraska Research Initiative.

*The $e$-Xtra logo stands for "electronic extra" and indicates there are supplementary materials published online.

The author(s) declare no conflict of interest.

(c) 2021 The American Phytopathological Society factors in resistance development. Risk of resistance development depends on both the pathogen and fungicide in question (Brent and Hollomon 2007). Pathogen risk factors include generation time, dispersal mechanism, and reproduction type. Fungicide risk factors include mode of action, number of applications per season, and dose.

Pathogen populations may be exposed to a range of fungicide doses in the field. Reasons for this include incomplete penetration of fungicides in the plant canopy, drift or run-off of fungicides, and dilution of fungicides inside the plant tissues. Most studies have focused on the role of fungicide dose in selection for resistant alleles (Lucas et al. 2015; van den Bosch et al. 2011). These studies suggest using sublethal fungicide doses to manage resistance because the selection pressure will remain low. Nevertheless, sublethal doses may stress the pathogen and increase its mutation rate, thus accelerating the emergence of mutations conferring resistance (Beckerman et al. 2015; Gressel 2011). Stress can increase mutational frequency by direct and indirect mechanisms (Galhardo et al. 2007; Koshiji et al. 2005; Parker and von Borstel 1987; Shor et al. 2013). For example, osmotic stress can damage DNA by inducing DNA breaks (Parker and von Borstel 1987), while proteotoxic stress can result in reduced expression of the DNA repair pathway genes (Shor et al. 2013). Studies in human fungal pathogens have shown that sublethal antifungal stress increases the rate of point mutations (Avramovska and Hickman 2019), aneuploidy, and chromosomal rearrangements (Avramovska and Hickman 2019; Harrison et al. 2014; Shapiro 2015; Shor and Perlin 2015). But studies on fungal plant pathogens give an unclear picture of the role of sublethal fungicide dose in increasing mutation rate (Ajouz et al. 2010; Amaradasa and Everhart 2016; Chen et al. 2015; Dowling et al. 2016; Schnabel et al. 2014; Troncoso-Rojas et al. 2013). Botrytis cinerea strains exposed to iprodione for 20 generations did not show any changes in the allele size at the nine simple sequence repeat (SSR) loci tested (Ajouz et al. 2010). 
However, isothiocyanate exposure induced random mutations in inter-simple sequence repeats regions of five Alternaria alternata strains with variable mutational frequency among strains (Troncoso-Rojas et al. 2013). In two out of three strains of Monilinia fructicola, gain or loss of the transposable element $\mathrm{Mftcl}$ was observed after in vitro exposure to azoxystrobin or a mixture treatment of azoxystrobin and SYP-Z048 (Chen et al. 2015). But field populations of $M$. fructicola neither showed any changes in their SSR profile nor in the translocation of the $M f t c l$ transposon after sublethal exposure to azoxystrobin or propiconazole. Collectively, these studies suggest that the effect of fungicide stress on mutational frequency is unclear and the effect varies among strains of the same species (Amaradasa and Everhart 2016; Chen et al. 2015; Troncoso-Rojas et al. 2013) and among exposure to different fungicides (Amaradasa and Everhart 2016; Chen et al. 2015).

One limitation of these studies was the use of genetic markers to determine the effect of fungicide exposure on mutational frequencies, which could only assess the impact of fungicide stress on a small fraction of the genome. Whole genome sequencing (WGS) studies show that plant pathogens have extensive genome plasticity (Miller et. al 2018; Moolhuijzen et al. 2018; O'Sullivan et al. 1998) and stresses induce different types of genomic perturbations. Hostinduced stress increases chromosomal instability, copper and potassium chlorate stresses affect transposable element (TE) movement, while heat stress induces chromosomal instability as well as TE movement in plant pathogens (Anaya and Roncero 1996; Chadha and Sharma 2014; Kasuga et al. 2016; Kistler and Miao 1992; Möller et al. 2018). Fungicide stress-induced genomic instability has not been studied previously in plant pathogens. The complete mutational profile of a fungicide-exposed strain may depend on the mode of action of a fungicide, which determines the type of stress it imposes. For example, fungicides that inhibit osmotic signal transduction can cause osmotic stress and induce DNA breaks (Parker and von Borstel 1987). Bias for different types of mutations such as single nucleotide polymorphisms (SNPs), insertions/deletions (INDELs), TE movement, and copy number variants (CNVs) are known to vary among stresses (Anaya and Roncero 1996; Chadha and Sharma 2014; Maharjan and Ferenci 2017). WGS can help us to elucidate the effect of fungicides with different modes of action on these different types of genomic variants. Additionally, the interaction effect of genomic background with fungicide stress exposure has been observed (Amaradasa and Everhart 2016; Chen et al. 2015; Troncoso-Rojas et al. 2013), though it has not been formally investigated. Studying fungicide stress on strains with diverse genomic backgrounds can give insight into the population-level dynamics of fungicide stress.

Sclerotinia sclerotiorum is an important pathogen that causes disease on $>400$ plant species (Boland and Hall 1994) and fungicides are commonly used for disease management. Fungicide resistance in $S$. sclerotiorum has been reported for benomyl, carbendazim, thiophanate-methyl (microtubulin synthesis inhibitors; Attanayake et al. 2013; Gossen et al. 2001; Lehner et al. 2015; Ma et al. 2009; Penaud et al. 2003), azoxystrobin, pyraclostrobin (respiration inhibitors; Tóthová et al. 2019), and iprodione (osmotic signal transduction inhibitor; Molaei et al. 2020). In a previous study, nine $S$. sclerotiorum strains were exposed to sublethal doses of five fungicides: azoxystrobin, boscalid, iprodione, pyraclostrobin, and thiophanate-methyl for 12 generations with experimental replication (Amaradasa and Everhart 2016). SSR analysis of all the progenitor and fungicide-exposed strains showed that 12 of 85 fungicide-exposed strains were mutated. The goal of the present study was to inspect genome-wide signatures of sublethal fungicide stress in strains of $S$. sclerotiorum with different genomic backgrounds. To accomplish this goal, a subset of strains from the previous study were selected for WGS. To study the effect of genomic background on the mutational repertoire, multiple progenitor and derived strains were sequenced.
The genomic features of $S$. sclerotiorum made it a suitable model system for conducting this study. The small genome size of S. sclerotiorum (38.8 Mb) enabled us to sequence more number of strains cost-effectively than would be possible with a fungus with a larger genome. Genome stability $(12.96 \%$ TE content; Derbyshire et al. 2017) provided less chances of background mutations as compared with fungal genomes with higher TE content. The $S$. sclerotiorum genome is also optically mapped (assembled to chromosomal level) and annotated (Derbyshire et al. 2017), which helped us to make conclusions on a per chromosome-basis. As genomic perturbations can have deleterious effects on strain fitness or aggressiveness (Jeon et al. 2013), investigating the consequences of genomic alterations on phenotype can give an insight into the feasibility of genome plasticity in natural environments. The haploid nature of the $S$. sclerotiorum genome enabled elucidation of the phenotypic effect of mutations on strain aggressiveness without concern of dominant alleles masking the recessive alleles.

To characterize genomic effects of sublethal fungicide stress in $S$. sclerotiorum and study the role of different genomic backgrounds in generating diversity, our objectives were to (i) determine mutation frequency in control and fungicide-exposed strains; (ii) characterize mutations as SNPs, INDELs, CNVs, and TE insertions; (iii) determine the genomic distribution of mutations; (iv) characterize variation among genomic backgrounds; and (v) determine the fitness consequences of genome perturbations. We sequenced a subset of $S$. sclerotiorum genomes that were exposed to sublethal fungicide stress previously (Amaradasa and Everhart 2016) and determined the number, type, and distribution of mutations with respect to the progenitor strains. To characterize the variation in the genomic backgrounds of the progenitor strains, genomic variants were determined with respect to the reference genome. Finally, the phenotypic effect of mutation accumulation on strain aggressiveness was studied to determine the fitness effects of the genome-wide mutations. This is the first study to attempt to characterize genomic signatures of sublethal fungicide stress in a plant pathogen and study the role of within-species diversity on the mutational repertoire produced in response to fungicide stress. Understanding factors that increase the mutation rate and accelerate resistance emergence can help to devise disease management strategies that delay resistance evolution and prolong the life of currently used fungicides.

\section{MATERIALS AND METHODS}

Strains and fungicides. Five $S$. sclerotiorum strains (Table 1; strain IDs $152,467,555,594$, and 646) were vegetatively cultured under different treatment conditions to obtain 50 experimentally evolved strains as described in Amaradasa and Everhart (2016). In brief, each progenitor strain was subcultured independently on sublethal doses of azoxystrobin, boscalid, iprodione, thiophanatemethyl, and as a negative control to obtain five experimentally evolved strains and the experiment was repeated. Sublethal fungicide exposure was achieved by growing strains on a concentration gradient of fungicide and then collecting mycelia from the sublethal

TABLE 1. Sclerotinia sclerotiorum strains used in the current study

\begin{tabular}{lccccc}
\hline Strain & Origin & Year & Aggressiveness $^{\mathrm{a}}$ & MCG $^{\mathrm{b}}$ & Host cultivar $^{\mathrm{c}}$ \\
\hline 152 & Nebraska & 1980 & 3.9 & 4 & Great Northern \\
467 & Colorado & 1996 & 4.6 & 45 & Pinto \\
555 & Minnesota & 2004 & 6.4 & 44 & Bunsi \\
594 & California & 2004 & 4.6 & 21 & Bunsi \\
646 & Washington & 2005 & 5.4 & 60 & Bunsi \\
\hline
\end{tabular}

a Aggressiveness was rated on the modified Petzoldt and Dickson scale of 1 to 9 (Terán et al. 2006). A moderately resistant dry bean cultivar, G122, was used for evaluation.

b MCG, mycelial compatibility group.

c Host cultivar of common bean (Phaseolus vulgaris) from which these strains were collected 
exposure region (50 to $100 \%$ inhibition zone). The amount of collected mycelia was increased by growing it on unamended potato dextrose agar (PDA) so that it could be subjected to another round of sublethal fungicide exposure. The process of sublethal fungicide exposure and inoculum multiplication on unamended media were important for minimizing the effect of selection and were repeated a total of 12 times. At the end of the two experiments, 10 subcultured strains were derived from each progenitor. The sclerotia of all the strains were stored at $4{ }^{\circ} \mathrm{C}$ until further use. For the present study, strains were revived from sclerotia by plating them on $1.5 \%$ water agar at room temperature. After 5 to 6 days, a 6-mm plug was excised from the actively growing margin of the mycelial colony and placed upside-down on PDA plates covered with cellophane to facilitate mycelial collection for DNA extraction.

DNA extraction and WGS. DNA was purified from 800 to $1,000 \mathrm{mg}$ of actively growing mycelia of 55 strains that were scraped from 2- to 3-day-old cellophane PDA plates. Mycelia were ground with liquid nitrogen in presterilized pestle and mortar. For DNA extraction, DNeasy Plant Maxi Kit (Qiagen, Hilden, Germany) was used according to the manufacturer's instructions and DNA was stored at $-20^{\circ} \mathrm{C}$ until further use. About 1.3 to $8.8 \mu \mathrm{g}$ of DNA per sample was shipped on dry ice to the Philadelphia, PA receiving center for Beijing Genomics Institute (BGI, China). The samples were further shipped on dry ice to the BGI laboratory in Hong Kong. Before library preparation, DNA concentration was checked using Qubit 3 Fluorometer (Invitrogen, Carlsbad, CA), which ranged from 8.4 to $86.8 \mathrm{ng} / \mu \mathrm{l}$, and DNA integrity was evaluated using gel electrophoresis on a $2 \%$ agarose gel. High quality DNA was subjected to WGS in one lane of Illumina HiSeq 4000 with 150-base paired-end reads and 350-bp insert size.

Data filtering. Sixteen GB of raw data in fastq format with adapters being trimmed were received from the sequencing facility. Quality of raw read pairs was assessed using FastQC version 0.11 (Andrews 2010) and sequences below $99.84 \%$ accuracy (phredscaled quality threshold of 28) were trimmed using trimmomatic version 0.36 (Bolger et al. 2014). Fifty-three million reads were aligned to the $S$. sclerotiorum reference genome version 2 (Derbyshire et al. 2017) using Bowtie version 2.2 (Langmead and Salzberg 2012) setting the maximum insert size parameter to 800 bp. SAMtools version 1.3 ( $\mathrm{Li}$ et al. 2009) was used to convert SAM files into BAM format and to index and sort the BAM files. Optical duplicates were filtered using Picard version 2.9 (Broad Institute 2017). Different variant types were called using different software on networked computers provided by the Holland Computing Center at the University of Nebraska-Lincoln.

Characterization of SNPs and INDELs. SNP and INDEL variants were called using HaplotypeCaller and GenotypeGVCFs in GATK version 3.4 (McKenna et al. 2010) and a single variant call format (VCF) file for all the strains was created for further analysis. All components of the reference guided assembly pipeline and variant calling using GATK were implemented in a makefile available at https://github.com/everhartlab/read-processing. Quality variants were filtered using the package vcfR version 1.7 (Knaus and Grünwald 2017) implemented in R version 3.6.2 (R Core Team 2019). The minimum mapping quality threshold was set to a score of 41 on phred-scale ( $>99.99 \%$ accuracy). To determine the effect of sublethal fungicide exposure in derived strains with respect to each progenitor strain within an experiment, only loci with $>5 \times$ coverage for all strains in the comparison group were retained. Strain 594 exposed to iprodione in the first experiment appeared to be mislabeled or contaminated based on its pattern of variants and was not included in further analyses. For determining the genomic differences of each progenitor strain with respect to the reference genome, only loci with $>5 \times$ coverage for at least three out of the five strains were retained. Loci with $\leq 5 \times$ coverage were removed from the analysis.

Variants identified from GATK were characterized as SNPs if one nucleotide was substituted and were characterized as INDELs if one or more nucleotides were added or deleted compared with the sequence of the progenitor strain. SNPs were further classified as transitions or transversions. Positions of mutated loci were mapped on chromosomes and their occurrence in coding (exonic) or noncoding (intronic, intergenic) regions was determined.

Variants in tandem repeats and TEs were identified and discarded because of low confidence of variants called in repetitive sequences. Tandem repeats of more than $50 \mathrm{bp}$ length were identified using MUMmer version 4.0 (Marçais et al. 2018). Consensus sequences of TEs previously identified in the $S$. sclerotiorum genome were obtained from RepetDB (Amselem et al. 2019) and were BLASTed against the reference genome using BLAST version 2.7 (Altschul et al. 1990) to obtain the respective TE coordinates. A stringent e-value of $1 \times 10^{-30}$ and $85 \%$ identity were chosen as the cut-off. Variants in tandem repeats and TEs were identified and discarded using the packages vcfR version 1.7 (Knaus and Grünwald 2017), dplyr version 0.8.5 (Wickham et al. 2020), and tidyr version 1.1.0 (Wickham and Henry 2020) in R version 3.6.2 (R Core Team 2019).

Characterization of CNVs. CNVs, i.e., duplications and deletions $\geq 1,500 \mathrm{bp}$, were characterized with CNVnator version 0.4 (Abyzov et al. 2011) using a bin size of $300 \mathrm{bp}$. Bin sizes of 100 to $500 \mathrm{bp}$ were tested in $100 \mathrm{bp}$ increments for all progenitor strains and the optimum bin size was selected when the ratio of average read depth to its standard deviation was between 4 to 5 (as recommended by the software developers). The vcf file generated from CNVnator was imported into $\mathrm{R}$ version 3.6.2 ( $\mathrm{R}$ Core Team 2019) and further analysis was done using vcfR version 1.7 (Knaus and Grünwald 2017) and dplyr version 0.8.5 (Wickham et al. 2020). Quality filtering was done by removing CNVs with e-value (e-val1) $\geq 0.1$ and putative repetitive regions $(\mathrm{q} 0 \geq 0.5 ; \mathrm{CNVs}$ with $\geq 50 \%$ reads mapped with zero mapping quality). For determining the effect of sublethal dose exposure, $\mathrm{CNVs}$ in the progenitor strains that overlapped with the corresponding derived strains were removed.

To determine aneuploidy, putative repetitive regions were retained. A strain was said to possess an extra copy of a given chromosome if $>85 \%$ of the chromosome was duplicated. If duplications or deletions constituted 40 to $85 \%$ of the chromosome, it was considered as partial gain or loss of the chromosome.

Characterization of TE insertions. Consensus TE fasta sequences (178) from the $S$. sclerotiorum genome (Amselem et al. 2011) were obtained from RepetDB (Amselem et al. 2019). Thirtysix of these TE sequences belonged to Class I transposons, 71 TE sequences belonged to Class II transposons, and 71 TE sequences were unclassified. The consensus sequences were used as an input in RetroSeq (Keane et al. 2013) to detect TE insertions in the progenitor and derived strains. VCF files of TE insertions were obtained as the output, which were quality filtered according to the number of supporting reads (GQ), confidence on the breakpoint (FL; range from 1 to 8 ), and proximity to TEs in the reference genome. TE insertions with FL $\geq 6$ were kept. Further, if the FL was 6 , variants with a minimum GQ of 28 were retained and for higher FL values, variants with a minimum GQ of 20 were retained.

Downstream analyses. To study the effect of fungicide exposure on frequency, type, and distribution of mutations, variants that were previously called with respect to the reference genome were filtered such that only those variants were retained that mutated in the derived strains with respect to the progenitor strains. This filtering step was done in $\mathrm{R}$ version 3.6.2 (R Core Team 2019) using vcfR version 1.7 (Knaus and Grünwald 2017) and dplyr version 0.8.5 (Wickham et al. 2020). The number of mutations in control and fungicide-exposed strains (derived from the same progenitor strain) were tested for statistically significant differences with respect to coding and noncoding regions, type of mutation (SNP, INDEL, CNV, or TE insertion), type of SNP (transition or transversion), and the chromosome on which the mutations were found. To determine the variation among the progenitor strain genomes, the number of point mutations (SNPs and INDELs), 
CNVs, and TE insertions called with respect to the reference genome were tested for statistical significance. A goodness of fit $\chi^{2}$ test was performed when the number of observations were more than five and an exact binomial test was performed otherwise at $\alpha=$ 0.05 in $\mathrm{R}$ version 3.6.2 ( $\mathrm{R}$ Core Team 2019).

To identify mutational hotspots, the genome was divided into nonoverlapping bins of lengths 500 and $10,000 \mathrm{bp}$, and the number of point mutations in each bin was determined. A bin was considered a mutational hotspot when the number of mutations in the bin was an outlier as determined by boxplot statistics (more than the 3rd quartile +1.5 times the interquartile range). A mutational hotspot was considered a treatment effect when the number of mutations in the corresponding control were less than or equal to the 3rd quartile. The putative functions of genes in the mutational hotspots were identified using the Batch CD-Search tool (MarchlerBauer and Bryant 2004; Marchler-Bauer et al. 2011).

Change in strain aggressiveness. High number of accumulated mutations resulting from fungicide stress may have a negative effect on the fitness of haploid S. sclerotiorum strains. To study the effect of mutation accumulation on strain aggressiveness, straw test was conducted on a moderately resistant dry bean genotype, G122, using the methodology described by Otto-Hanson et al. (2011).

Sclerotia were surface sterilized with a solution of $50 \%$ Clorox bleach and $50 \% \mathrm{dH}_{2} \mathrm{O}$ followed by rinsing twice with $\mathrm{dH}_{2} \mathrm{O}$ for 3 min each. Sterile sclerotia were dried on sterile paper towels for 20 to $30 \mathrm{~s}$, plated on $1.5 \%$ water agar, and stored at room temperature to reactivate the sclerotia. After 5 to 6 days, a $6 \mathrm{~mm}$ plug was taken from the growing mycelial edge and transferred to PDA. Two-dayold PDA cultures were used for inoculating dry bean plants. Sclerotia from two strains could not be revived: iprodione exposed strain 467 in the first experiment and boscalid exposed strain 594 in the second experiment. Aggressiveness assays were performed as two separate experiments set up in completely randomized design with four replications. Dry bean plants were inoculated 21 days after germination using straws with mycelial plugs. Clear drinking straws were cut into pieces of $2.5 \mathrm{~cm}$ length each that were sealed at one end. Straw pieces were filled with two mycelial plugs excised from the PDA cultures such that the mycelial surface faced the open end of the straw piece. Stem was cut at $2.5 \mathrm{~cm}$ above the fourth node and covered with the straw piece containing inoculum. Plants were incubated at $26 \pm 2{ }^{\circ} \mathrm{C}$ day and $20 \pm 2^{\circ} \mathrm{C}$ night temperatures in the greenhouse for 8 days and were rated using the modified Petzoldt and Dickson scale ranging from 1 to 9 (Terán et al. 2006). Data from the two greenhouse experiments were combined. Statistical analysis of the mean aggressiveness score of progenitor and derived strains was done using Kruskal-Wallis rank sum test at $\alpha=0.05$ in $\mathrm{R}$ version 3.6.2 (R Core Team 2019).

\section{RESULTS}

Genome alignment statistics. We sequenced a total of 55 strains: five progenitor, 10 control (unexposed), and 40 fungicideexposed strains, of which one fungicide-exposed strain was removed from the analysis due to contamination. Read mapping to the reference genome resulted in fair to high read depths and coverage for both nuclear and mitochondrial genomes. The average read depths of nuclear and mitochondrial genomes were $16.47 \times$ and $199.64 \times$, respectively, and the average coverage was 98.5 and $99.6 \%$, respectively.

Frequency and characterization of mutations. To investigate the effect of fungicide exposure and genomic background on the rate of spontaneous mutations, the number of SNPs, INDELs, CNVs, and TE insertions were determined for all the derived strains. The type of point mutation varied according to the genomic background of the strain (Table 2). SNPs were more frequent than INDELs in all strains and accounted for $60.74 \%$ of point mutations in strain 594 and $84.52 \%$ of point mutations in strain 467 . Transitions were more frequent than transversions and accounted for $55 \%$ of SNPs in strain 467 and $80.94 \%$ of SNPs in strain 594.

On average, the frequency of INDELs was higher in fungicideexposed strains than their control (unexposed) counterparts in both experiments (Fig. 1). INDEL frequency was significantly higher in strain 555 exposed to azoxystrobin and iprodione in the first experiment (Fig. 1; $P \leq 0.05$ ). The number and type of SNPs (transition or transversion) were not affected by fungicide exposure (Supplementary Fig. S1; $P>0.05$ ). G $>$ A (or A $>$ G) transitions were the most common among all strains followed by $\mathrm{C}>\mathrm{T}$ (or $\mathrm{T}>$ C) transitions, but the frequency of different types of transitions and transversions were not significantly affected by fungicide exposure (Supplementary Fig. S2; $P>0.05$ ). Collectively, strain 555 independently exposed to iprodione in the first experiment had a significantly higher number of point mutations than the corresponding control (Supplementary Fig. S3; $P \leq 0.05$ ).

The number of CNVs in derived strains did not show consistent patterns among experiments suggesting that fungicide exposure did not affect CNV frequency and that random CNVs were common in the $S$. sclerotiorum genome (Supplementary Fig. S4). The progenitor strain 152 had aneuploidy of chromosome 7, which was retained partially or completely in $30 \%$ of its derived strains. Other progenitor strains did not show aneuploidy but $18 \%$ of their derived strains partially or completely gained an extra copy of chromosome 7 (Supplementary Table S1).

Among classifiable TE insertions, the greatest number of TE insertions in all genomic backgrounds were terminal inverted repeats (TIR) (Supplementary Fig. S5). The number of TE insertions varied according to the fungicide-strain combination (Fig. 2). On average, strains 467, 555, and 646 had a lower number of TE insertions after fungicide exposure compared with the control, except strain 646 exposed to iprodione in the second experiment (Fig. 2). Strain 152 had a higher number of TE insertions than the control in azoxystrobin exposed strains, which was significant in the second experiment $(P \leq 0.05)$.

To characterize the differences among the genomic backgrounds of the progenitors, point mutations, TE insertions, and CNVs were characterized relative to the reference genome. The progenitor strain 555 had the highest number of point mutations, TE insertions, and CNVs, suggesting that it has maximum divergence from the reference genome (Fig. 3). The progenitor strains 152 and 467 did not differ significantly among each other in the number of point mutations, TE insertions, and CNVs (Fig. 3; $P>0.05$ ).

Overall, the genomic background had a prominent effect on the frequency of SNPs, INDELs, and TE insertions, and fungicide exposure affected point mutations and TE insertions in certain fungicide-strain combinations.

Genomic distribution of mutations. Bias in the genomic distribution of point mutations was studied on a per chromosomebasis, in nonoverlapping bins of sizes 500 and 10,000 bp, and in the coding and noncoding regions. Our results showed that the genomic background affected both the accumulation of mutations on chromosomes (Fig. 4), in 10,000 bp bins (Table 3), and in coding versus noncoding regions (Table 2). Irrespective of fungicide exposure, mutational hotspots were identified on chromosome 15 for strains 152, 467, and 594, chromosome 11 for strains 555, 594,

TABLE 2. Percentage (mean and standard errors) of point mutations (single nucleotide polymorphisms [SNPs] and insertions/deletions) accumulated across control strains in two experiments

\begin{tabular}{lccl}
\hline Strain & Coding region $(\%)$ & SNP $(\%)$ & Transition $(\%)$ \\
\hline 152 & $63.89 \pm 13.89$ & $80.56 \pm 2.78$ & $75.71 \pm 4.29$ \\
467 & $83.33 \pm 16.67$ & $84.52 \pm 1.19$ & $55.00 \pm 5.00$ \\
555 & $38.83 \pm 0.19$ & $76.69 \pm 6.24$ & $78.42 \pm 1.00$ \\
594 & $34.44 \pm 12.22$ & $60.74 \pm 12.6$ & $80.94 \pm 0.67$ \\
646 & $27.08 \pm 2.08$ & $62.5 \pm 4.17$ & $74.11 \pm 11.61$ \\
\hline
\end{tabular}



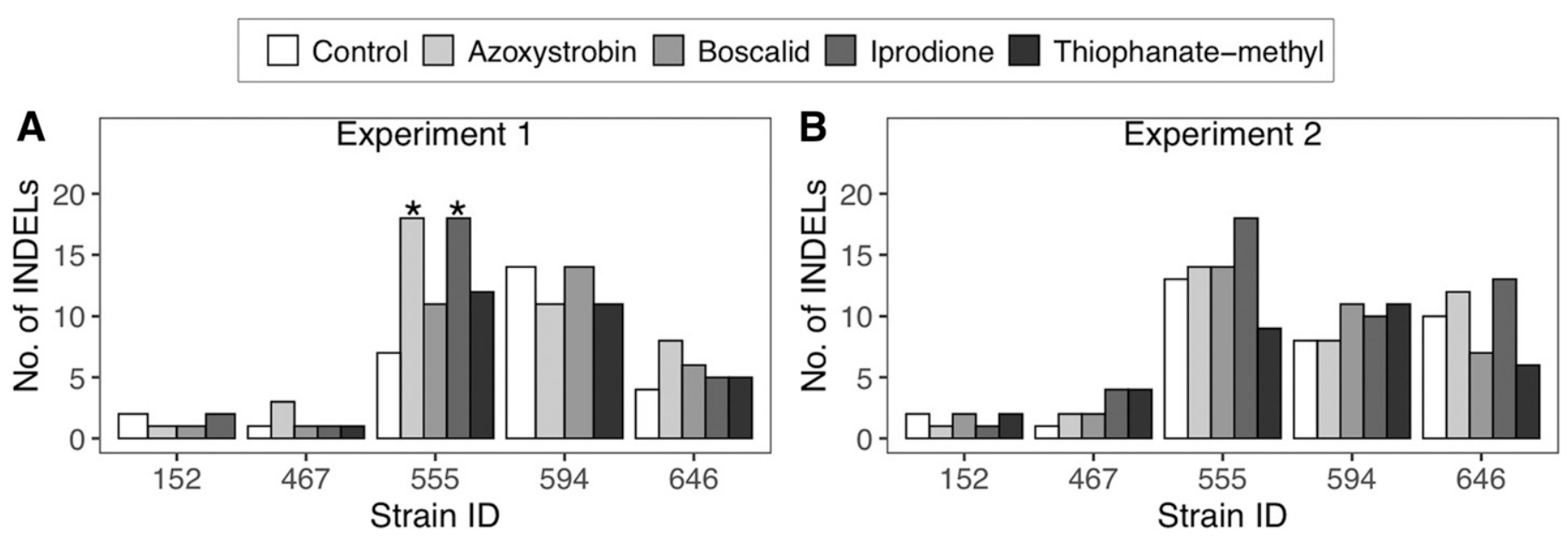

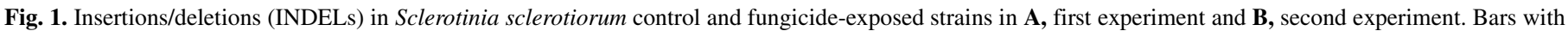

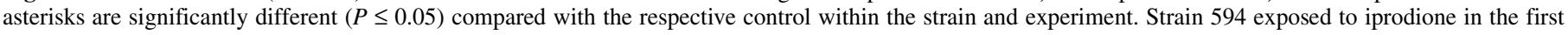
experiment was removed from the analysis because it was contaminated.
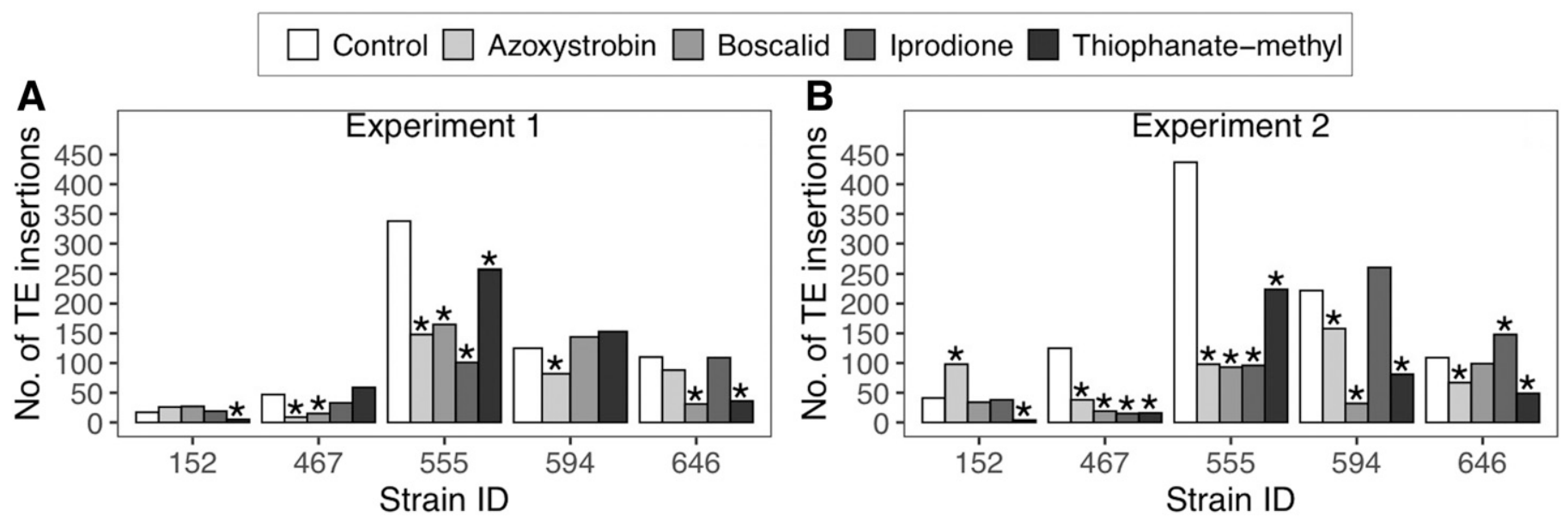

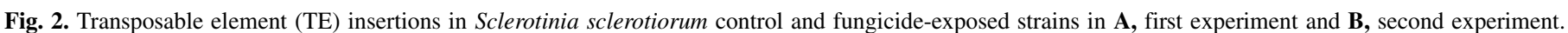

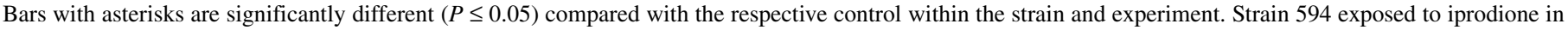
the first experiment was removed from the analysis because it was contaminated.
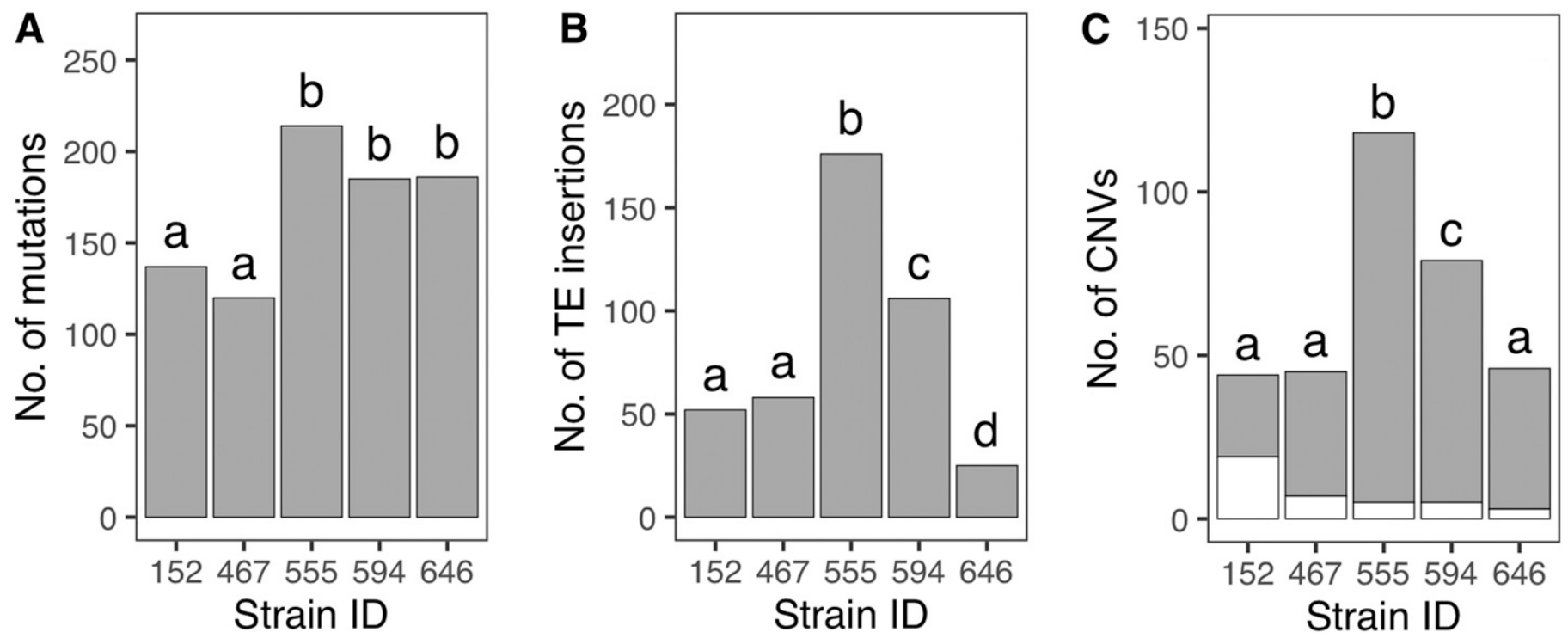

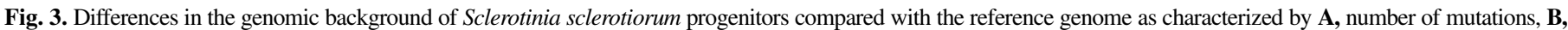

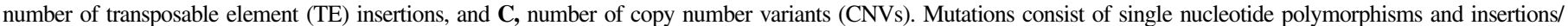

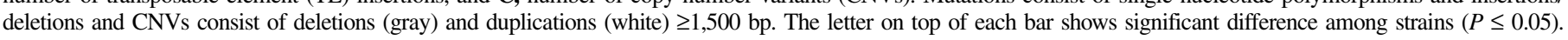


and 646, chromosome 4 for strain 555, chromosome 12 for strain 594, and chromosome 2 for strain 646 (Fig. 5). When investigated in $10,000 \mathrm{bp}$ bins, mutational hotspots of size ranging from 1,214 to 2,590 bp were identified that harbored eight genes (Table 3). The gene sscle_15g107310 was classified as a heavy metal translocating P-type ATPase, which transports or detoxifies heavy metals and the gene sscle_12g089740 as an MC/SLC25 family protein, which transfers molecules across mitochondrial membranes. The gene sscle_04g033710 had a protein kinases (PKc_like super family) conserved domain, while the other genes did not have known conserved domains. The mutational hotspots (Table 3 ) had a total of 76 point mutations, 73 of which were SNPs. Out of 73 SNPs, G > A (or $\mathrm{A}>\mathrm{G}$ ) transitions accounted for $50.68 \%$ of SNPs and $\mathrm{C}>\mathrm{T}$ (or $\mathrm{T}>\mathrm{C}$ ) transitions accounted for $34.24 \%$ of SNPs. The mean GC content of these hotspots was $43.9 \%$ (range: 38.18 to $45.35 \%$ ). Genomic background also affected the accumulation of point mutations in coding versus noncoding regions (Table 2). Strain 467 had $83.33 \%$ of point mutations in the coding region, while strain 646 only had $27.08 \%$ of point mutations in the coding region.

Fungicide exposure affected the genomic distribution of mutations in certain strain-fungicide combinations. Chromosome 11 had a significantly greater number of INDELs in strain 555 exposed to thiophanate-methyl in the first experiment and azoxystrobin in the second experiment than the respective controls $(P \leq 0.05)$. On average, the number of point mutations in the noncoding regions of fungicide-exposed strain 555 was higher than the control (Supplementary Fig. S6) and iprodione exposure of this strain in the first experiment resulted in a significantly higher mutation frequency $(P \leq 0.05)$. Twenty-one genes only mutated in fungicide-exposed strains (Supplementary Table S2), out of which two genes mutated in more than three fungicide-exposed strains. The gene sscle_11g081320 mutated in strain 555 independently exposed to boscalid, iprodione, and thiophanate-methyl in the first experiment and in strain 555 exposed to thiophanate-methyl in the second experiment. The gene sscle_14g101330 mutated in strain 555 independently exposed to azoxystrobin, iprodione, and thiophanate-methyl in the first experiment and in strain 555 independently exposed to iprodione and thiophanate-methyl in the second experiment. The sscle_11g081320 gene is a hypothetical protein with no conserved domains and sscle_14g101330 is a potential nucleoside hydrolase with a bacterial conserved domain of DNA polymerase III subunit gamma/tau.

Overall, the genomic background had a prominent effect on the genomic distribution of point mutations and fungicide exposure affected this distribution in certain strain-fungicide combinations.

Change in strain aggressiveness. Straw tests were conducted to examine any change in strain aggressiveness due to mutation accumulation. For all progenitor and derived strains, the aggressiveness varied from 3.38 to 6.00 (Supplementary Table S3). Among progenitor strains, the strain 555 was the most aggressive. Strain aggressiveness did not significantly change in control and exposed strains except in iprodione exposed strain 555 in the second experiment $(P \leq 0.05$; Table 4$)$. In general, mutation accumulation did not impact strain aggressiveness.
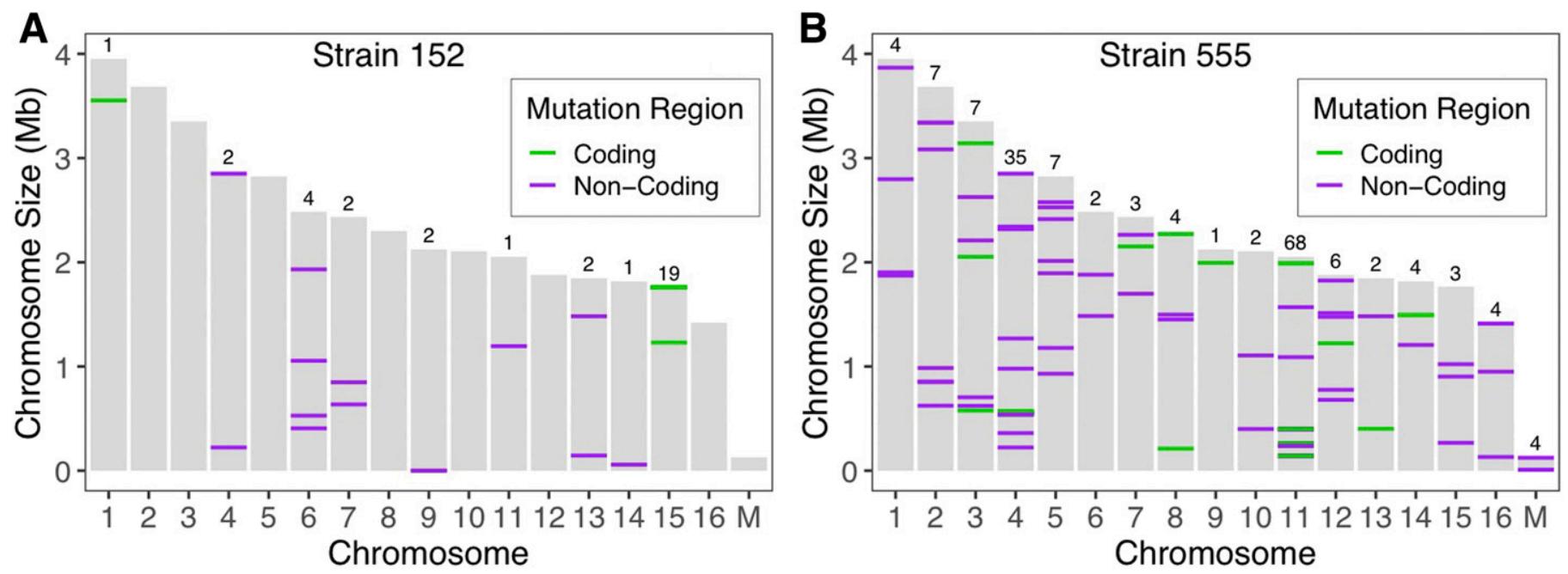

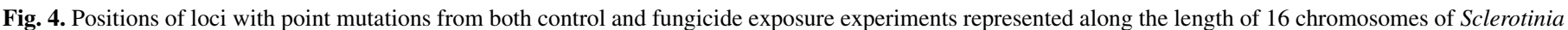

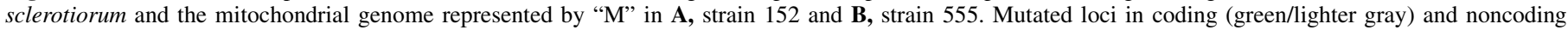

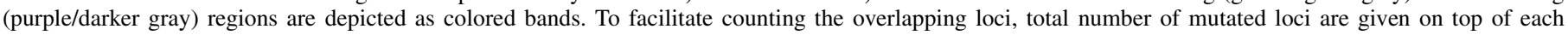
chromosome.

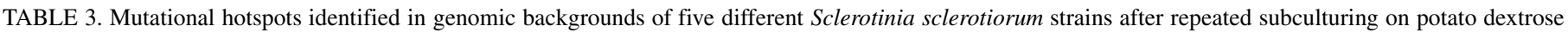
agar for 12 generations

\begin{tabular}{|c|c|c|c|c|c|c|}
\hline \multirow[b]{2}{*}{ Strain(s) } & \multirow[b]{2}{*}{ Chromosome } & \multicolumn{2}{|c|}{ Genome coordinates } & \multirow[b]{2}{*}{ Hotspot size (bp) } & \multirow[b]{2}{*}{ Gene(s) in hotspot } & \multirow[b]{2}{*}{ Number of mutations } \\
\hline & & Start & End & & & \\
\hline 152 & 15 & 1757488 & 1759192 & 1,704 & sscle_15g107310 & 10 \\
\hline 467 & & & & & & 9 \\
\hline \multirow[t]{2}{*}{555} & 4 & 540247 & 542175 & 1,928 & sscle_04g033580; sscle_04g033590 & 10 \\
\hline & & 565606 & 566820 & 1,214 & sscle_04g033700; sscle_04g033710 & 9 \\
\hline \multirow[t]{2}{*}{555} & 11 & 140120 & 141807 & 1,687 & sscle_11g081330 & 7 \\
\hline & & 238200 & 239741 & 1,541 & sscle_11g081640 & 4 \\
\hline 555 & 11 & 396918 & 399508 & 2,590 & - & 9 \\
\hline 594 & & & & & & 6 \\
\hline 646 & & & & & & 8 \\
\hline 594 & 12 & 1076943 & 1078608 & 1,665 & sscle_12g089740 & 10 \\
\hline
\end{tabular}




\section{DISCUSSION}

We found that sublethal fungicide exposure increased the mutation frequency in S. sclerotiorum in certain genomic backgrounds. This exposure significantly increased INDEL frequency in one genomic background (Fig. 1) and generally suppressed TE insertions (Fig. 2). A pronounced effect of the genomic background was observed on genome instability. In general, strain 555 had more propensity to create genetic variation, which is important for adapting to stressful environments and emergence of fungicide resistance. For possible intervention in resistance evolution, it is important to understand the factors that accelerate adaptation. This study suggests that sublethal fungicide doses can act as a genomic stressor in S. sclerotiorum and promote mutagenesis in certain genomic backgrounds, which could accelerate the emergence of alleles conferring fungicide resistance.

Several of the fungicide-exposed strains sequenced in the present study were shown previously to have mutations identified via SSR and amplified fragment length polymorphism (AFLP) genotyping (Amaradasa and Everhart 2016). Overall, SSR mutations were more frequent for strains exposed to iprodione and azoxystrobin, although strains 152, 467, and 555 only showed changes in AFLP profiles. WGS analysis conducted in the present study showed that the frequency of INDELs increased in almost all genomic backgrounds with a more prominent effect on strain 555 exposed to iprodione and azoxystrobin in the first experiment. Although searches of SSR loci were made using the WGS assemblies, no loci were identified (data not shown) and is likely due to the difficulty in assembling repetitive regions (Treangen and Salzberg 2012). Nevertheless, the increased point mutation frequency owing to fungicide stress was congruent with the SSR and AFLP results obtained in the previous study.

The relationship between TE insertion and stress has not been examined in S. sclerotiorum before. In other organisms, TEs are known to be activated or suppressed under stress and the consequences can vary with genomic background (Horváth et al. 2017). In the current study, TE insertion was mostly suppressed under sublethal fungicide stress in all genomic backgrounds except in azoxystrobin exposed strain 152 and iprodione exposed strain 646 (Fig. 2). Similar to these results, sublethal fungicide stress
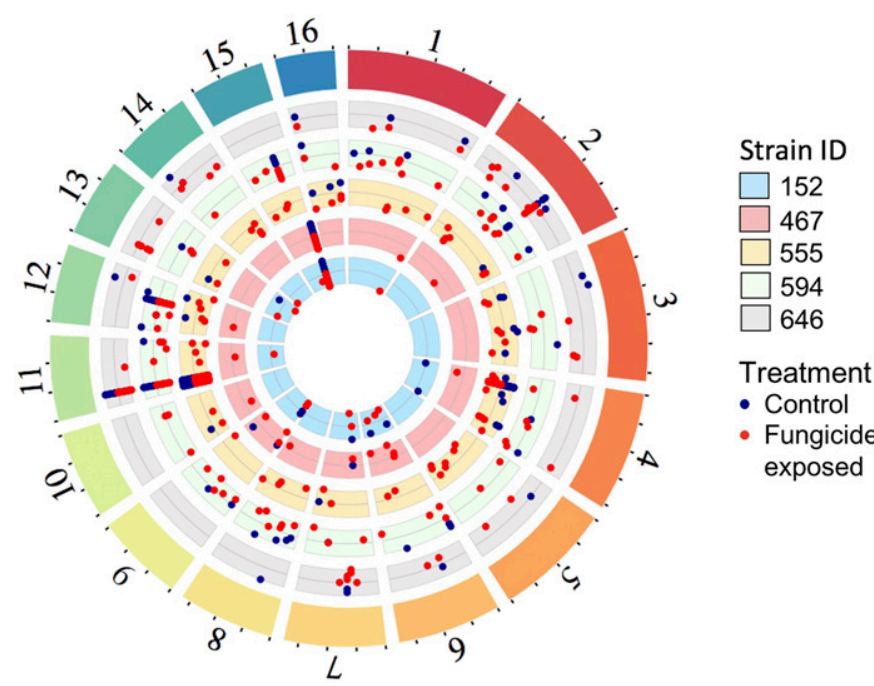

Fig. 5. Genomic distribution of point mutations in the control (dark blue) and the fungicide-exposed strains (red) on the 16 chromosomes of Sclerotinia sclerotiorum. Starting from the innermost circular track, the five genomic backgrounds are represented by different colored tracks; strain 152 (blue), 467 (pink), 555 (yellow), 594 (green), and 646 (gray). For each genomic background, point mutations in two control strains and eight fungicide-exposed strains are represented except for the genomic background of strain 594 where point mutations from seven fungicide-exposed strains are represented. activated TE movement in a strain by fungicide-dependent manner in M. fructicola (Chen et al. 2015).

The effects of fungicide exposure were evaluated across multiple strains originating from different states in the United States (Table 1), allowing additional insight into variation by genomic background. The point mutation frequency (Supplementary Fig. S3) and distribution (Figs. 4 and 5) among strains varied according to the genomic background, which suggests that considerable variation exists in the genome dynamics of $S$. sclerotiorum. Among strains evaluated in the present study, strain 555 had the highest frequency of point mutations in both nontreated controls and in fungicide exposure. This suggests that different strains likely have a different rate of mutation. Such difference may facilitate adaptation of S. sclerotiorum to stressful environments, therefore adaptation in the population may be driven by strains that are more prone to mutations. A study conducted in Candida albicans also found that the genomic background influences genomic stability and evolution (Gerstein and Berman 2020). Environmental and clinical fungal strains with an increased mutation rate due to faulty DNA repair machinery, called hypermutators, have been shown to adapt more rapidly to antifungal therapy and host stress (Boyce et al. 2017; dos Reis et al. 2019; Healey et al. 2016). Since genes involved in the DNA repair pathway are not fully characterized in most plant pathogens including S. sclerotiorum, it is unclear if the higher mutation rate observed in the strain 555 is due to defective DNA repair machinery.

In addition to the genomic plasticity of strain 555, an important difference was observed in the colony morphology of strain 555 . Mycelium in the colony was dark gray in color, which is likely due to increased melanin (Butler et al. 2009; Lazarovits et al. 2000). Melanin pigmentation plays diverse roles in fungi. It is a characteristic feature of fungi adapted to extreme heat, cold, $\mathrm{pH}$, oxidative stress, and radiation (Coleine et al. 2020; Gessler et al. 2014; Mironenko et al. 2000). Melanin protects the fungal cell from various stresses and has additional functions that are not fully understood (Eisenman et al. 2020). In the current study, we observed that the genome of the melanized fungal strain produced more mutations in response to fungicide stress, which could facilitate stress adaptation. Further studies are required to explore the relationship, if any, between melanization and stress-induced mutations.

Spontaneous mutations were observed in the control in the present study and similar results were obtained in experimental evolution studies conducted in Magnaporthe oryzae and Zymoseptoria tritici (Jeon et al. 2013; Möller et al. 2018). After serially transferring $M$. oryzae strains on artificial media up to 10 and 20 times, 200 to 350 point mutations were observed in the derived strains. Similar to the present study, a mutational bias was observed for SNPs over INDELs, transitions over transversions and a mutational bias was also observed for certain chromosomes. Virulence of $M$. oryzae decreased after 20 generations of serial transfer, however, strain aggressiveness in the present study did not change after mutation accumulation. The decrease in virulence of $M$. oryzae may be due to the deleterious effect of a higher number of mutations accumulated in the genome. The S. sclerotiorum haploid

TABLE 4. Aggressiveness (mean and standard errors) of Sclerotinia sclerotiorum strains at the beginning of the study and those with significant changes after experimentation

\begin{tabular}{llccc}
\hline Strain & Treatment & Experiment & \multicolumn{2}{c}{ Aggressiveness $^{\mathrm{a}}$} \\
\hline 152 & Progenitor & - & $4.25 \pm 0.16$ & - \\
467 & Progenitor & - & $4.63 \pm 0.26$ & - \\
555 & Progenitor & - & $5.38 \pm 0.26$ & - \\
& Iprodione & 2 & $3.38 \pm 0.56$ & $*$ \\
594 & Progenitor & - & $4.57 \pm 0.30$ & - \\
646 & Progenitor & - & $4.63 \pm 0.53$ & - \\
\hline
\end{tabular}

a Aggressiveness was rated on the modified Petzoldt and Dickson scale of 1 to 9 (Terán et al. 2006). A moderately resistant dry bean cultivar, G122, was used for evaluation. $* P \leq 0.05$ compared with progenitor. 
genome appears to be tolerant to perturbations, without a fitness cost, suggesting that plasticity may play an important role in adaptation of $S$. sclerotiorum to stresses like host defenses and unfavorable environmental conditions. The presence of genomic plasticity in this pathogen signifies that even during clonal propagation of S. sclerotiorum (Cubeta et al. 1997; Kamvar et al. 2017; Kohli and Kohn 1998), it can use several mechanisms to spontaneously increase genetic diversity.

Antifungal stress posed by azoles and echinocandins in human fungal pathogens is known to increase the rate of point mutations (Avramovska and Hickman 2019) but has a more prominent impact on aneuploidy and chromosomal rearrangements (Avramovska and Hickman 2019; Harrison et al. 2014; Shapiro 2015; Shor and Perlin 2015). A change in aneuploidy or CNVs in response to fungicide stress was not observed in the present study. However, fungicides tested did not belong to azoles and echinocandins, which might explain the difference in the observed genome dynamics. Irrespective of fungicide exposure, a high number of $\mathrm{CNVs}$, especially the propensity for large duplications on chromosome 7 and aneuploidy of this chromosome were observed in the S. sclerotiorum genome (Supplementary Table S1). Interestingly, this chromosome harbors regions with high density of repetitive sequences and repeat induced point mutations, which are associated with clusters of secreted and effector-like proteins (Derbyshire et al. 2017). Rapid gain and loss of the extra copy of this chromosome suggests that this strategy might be frequently used by $S$. sclerotiorum and may be helpful for host stress adaptation. Extensive CNVs were also observed during the vegetative growth of the haploid fungus $Z$. tritici (Möller et al. 2018), suggesting that chromosomal rearrangements might be a common mechanism of generating genetic variation in at least some plant pathogens.

According to the frequency of previous resistance reports in S. sclerotiorum (Attanayake et al. 2013; Gossen et al. 2001; Lehner et al. 2015; Ma et al. 2009; Molaei et al. 2020; Penaud et al. 2003; Tóthová et al. 2019), more mutations were expected after thiophanate-methyl exposure. However, out of the four fungicides tested, azoxystrobin and iprodione had more prominent effects on INDELs in strain 555 (Fig. 1) and TE insertions in strains 152 and 646 (Fig. 2). Although azoxystrobin and iprodione might be more stressful for S. sclerotiorum, high field usage of thiophanate-methyl to control $S$. sclerotiorum may be a more significant driver of fungicide resistance.
A few studies have tested the hypothesis of sublethal fungicide induced mutations in fungal pathogens in the same family as $S$. sclerotiorum. An in vitro study in M. fructicola showed that 8 of 15 SSR loci mutated in one of the three strains exposed to sublethal doses of azoxystrobin (Schnabel et al. 2014) and the movement of transposable element $M f t c l$ was affected by sublethal fungicide dose (Chen et al. 2015). In a follow-up study, field populations of M. fructicola were exposed to sublethal doses of azoxystrobin and propiconazole. The sensitivity of field populations did not change significantly, and mutations were not observed in the seven SSR loci tested (Dowling et al. 2016). This inconsistency may be either due to fungicide degradation and lack of exposure in the field setting or due to genomic changes not captured by the $7 \mathrm{SSR}$ loci. In another study, four $B$. cinerea strains were exposed to iprodione in vitro, which did not mutate at any of the nine SSR loci tested (Ajouz et al. 2010). However, such exposure changed the aggressiveness of the strains and led them to develop resistance to iprodione, fludioxonil, and dicloran fungicides. Collectively, these studies suggest that genetic markers may not provide sufficient information to study the effect of sublethal fungicide exposure. This is the first study to evaluate the role of sublethal fungicide stress in causing mutagenesis at the whole-genome level.

The approach used for sublethal fungicide exposure in the present study was designed such that the effect of fungicide stress on mutation emergence could be examined, while minimizing the effect of selection. To minimize the effect of selection, sublethal fungicide-exposed mycelia were collected and multiplied in the absence of fungicide, which was used for subsequent fungicide exposure. Fast-growing mycelial sectors were not used for subculturing. These fast-growing sectors might have had alleles conferring resistance or increased tolerance to the fungicide. This speculation is backed by a previous study where fungicide resistance was induced in the laboratory in S. sclerotiorum by exposing it to a sublethal fungicide concentration of fludioxonil and transferring the fast-growing sectors to a high fungicide concentration to select for resistance (Kuang et al. 2011). Among the 40 fungicide-exposed strains used in the present study, none of them developed fungicide resistance (Amaradasa and Everhart 2016). However, there was variation in the fungicide sensitivity of fungicide-exposed strains (Fig. 6). Fungicide sensitivity did not change significantly for 14 strains $(P>0.05)$, decreased significantly for 14 strains, and increased significantly for 12 strains

\section{Experiment $\bullet 1 \Delta 2 \quad$ Difference $\bullet \mathrm{ns} \bullet \mathrm{s}$}
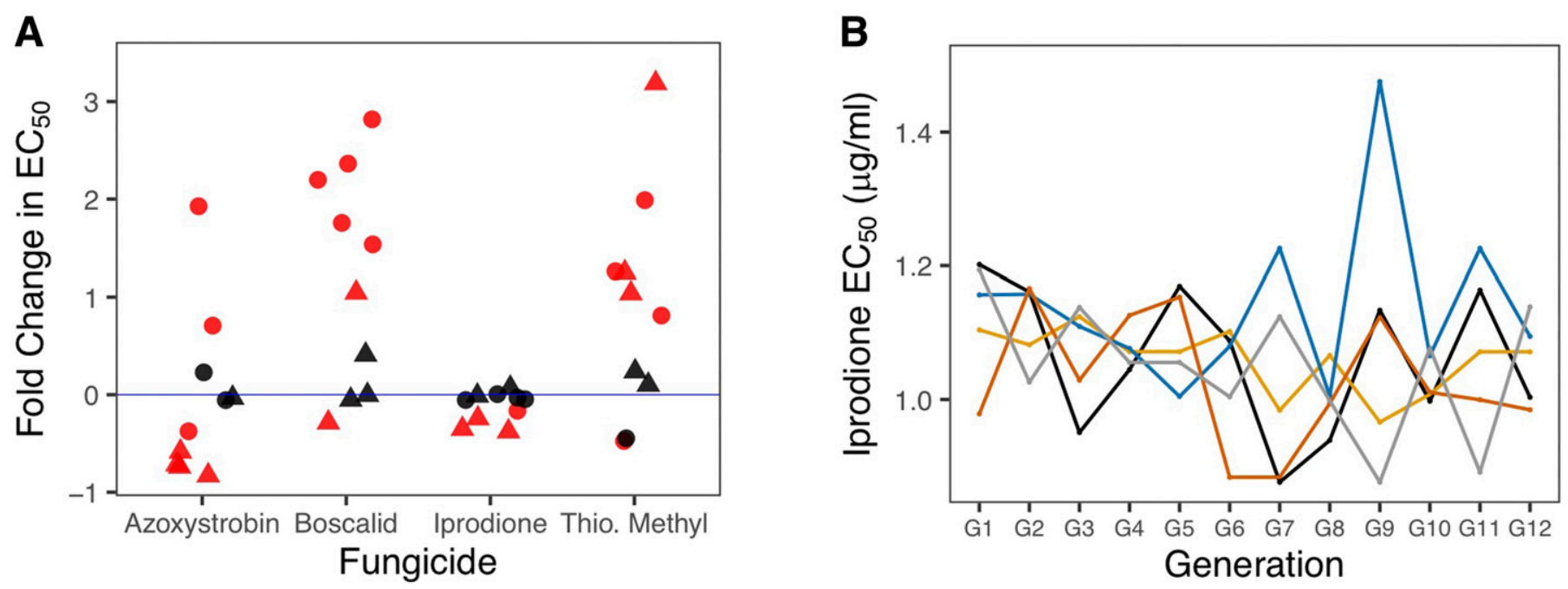

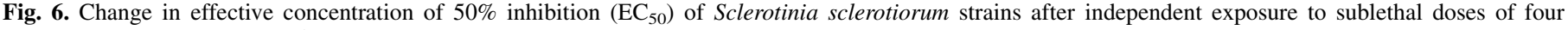

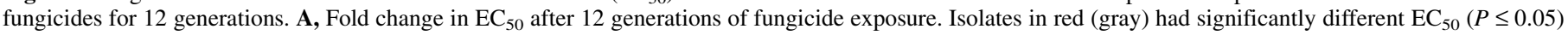

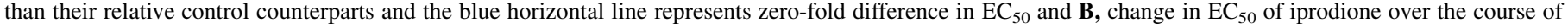
fungicide exposure. Fungicide sensitivity of progenitor was determined from results of G1 exposure. 
$(P \leq 0.05)$ (Amaradasa and Everhart 2016). Such random distribution of the sensitivity corroborates that the selection pressure was minimized during the experiment.

One limitation of the present study was that the genomic variation among the progenitor strains might not be completely represented by alignment to the available reference genome. Genomes of most of the progenitor strains varied considerably among each other and from the reference genome (Fig. 3). Strain 152 was derived from the reference genome strain, S. sclerotiorum 1980 UF-70, and hence showed few genomic aberrations than the reference genome. With the sequencing parameters used in the present study, high quality de novo assemblies were not achieved. However, the reference guided approach used in the present study yielded high quality variants. Future studies should use de novo genome assemblies to examine the novel genomic variation present in populations of $S$. sclerotiorum that may not otherwise be identified using referenceguided assembly alone.

Another limitation of this study was that the identified genomic variants were not validated experimentally. False-positive and falsenegative variants can result from sequencing errors, mapping errors, or erroneous detection by variant callers (Hwang et al. 2015). Although variants were quality filtered to obtain high confidence calls, the authors acknowledge that a small percentage of false-positive and false-negative variants may have been retained. Future studies should perform polymerase chain reaction or Sanger sequencing to validate variants of interest before performing further analyses.

Nonetheless, the present study shows that in vitro sublethal fungicide exposure can increase the mutation frequency in certain strains of $S$. sclerotiorum and strains with a highly mutable genomic background can generate a bigger allele pool that may hasten adaptation. A better understanding of the factors that accelerate resistance emergence is important to devise disease management strategies that delay resistance evolution and prolong the life of currently used fungicides.

\section{ACKNOWLEDGMENTS}

We thank Edgar Nieto Lopez for helping us in the strain aggressiveness assays.

\section{LITERATURE CITED}

Abyzov, A., Urban, A. E., Snyder, M., and Gerstein, M. 2011. CNVnator: An approach to discover, genotype, and characterize typical and atypical CNVs from family and population genome sequencing. Genome Res. 21:974-984.

Ajouz, S., Decognet, V., Nicot, P. C., and Bardin, M. 2010. Microsatellite stability in the plant pathogen Botrytis cinerea after exposure to different selective pressures. Fungal Biol. 114:949-954.

Altschul, S. F., Gish, W., Miller, W., Myers, E. W., and Lipman, D. J. 1990. Basic local alignment search tool. J. Mol. Biol. 215:403-410.

Amaradasa, B. S., and Everhart, S. E. 2016. Effects of sublethal fungicides on mutation rates and genomic variation in fungal plant pathogen, Sclerotinia sclerotiorum. PLoS One 11:e0168079.

Amselem, J., Cornut, G., Choisne, N., Alaux, M., Alfama-Depauw, F., Jamilloux, V., Maumus, F., Letellier, T., Luyten, I., Pommier, C., and Adam-Blondon, A. F. 2019. RepetDB: A unified resource for transposable element references. Mob. DNA 10:6.

Amselem, J., Cuomo, C. A., Van Kan, J. A., Viaud, M., Benito, E. P., Couloux, A., Coutinho, P. M., de Vries, R. P., Dyer, P. S., Fillinger, S., and Fournier, E. 2011. Genomic analysis of the necrotrophic fungal pathogens Sclerotinia sclerotiorum and Botrytis cinerea. PLoS Genet. 7:e1002230.

Anaya, N., and Roncero, M. I. G. 1996. Stress-induced rearrangement of Fusarium retrotransposon sequences. Mol. Gen. Genet. 253:89-94.

Andrews, S. 2010. FastQC: A quality control tool for high throughput sequence data. https://www.bioinformatics.babraham.ac.uk/projects/fastqc/

Attanayake, R. N., Carter, P. A., Jiang, D., del Río-Mendoza, L., and Chen, W. 2013. Sclerotinia sclerotiorum populations infecting canola from China and the United States are genetically and phenotypically distinct. Phytopathology 103:750-761.

Avramovska, O., and Hickman, M. A. 2019. The magnitude of Candida albicans stress-induced genome instability results from an interaction between ploidy and antifungal drugs. Genes Genom. Genet. 9:4019-4027.
Beckerman, J. L., Sundin, G. W., and Rosenberger, D. A. 2015. Do some IPM concepts contribute to the development of fungicide resistance? Lessons learned from the apple scab pathosystem in the United States. Pest Manag. Sci. 71:331-342.

Boland, G. J., and Hall, R. 1994. Index of plant hosts of Sclerotinia sclerotiorum. Can. J. Plant Pathol. 16:93-108.

Bolger, A. M., Usadel, B., and Lohse, M. 2014. Trimmomatic: A flexible trimmer for Illumina sequence data. Bioinformatics 30:2114-2120.

Boyce, K. J., Wang, Y., Verma, S., Shakya, V. P., Xue, C., and Idnurm, A. 2017. Mismatch repair of DNA replication errors contributes to microevolution in the pathogenic fungus Cryptococcus neoformans. MBio 8: e00595-e 17.

Brent, K. J., and Hollomon, D. W. 2007. Fungicide resistance in crop pathogens: How can it be managed? FRAC Monogr. No. 1, 2nd revised ed. Fungicide Resistance Action Committee, CropLife International, Brussels. https://www.frac.info/docs/default-source/publications/monographs/monograph-1.pdf

Broad Institute. 2017. Picard tools. http://broadinstitute.github.io/picard

Butler, M. J., Gardiner, R. B., and Day, A. W. 2009. Melanin synthesis by Sclerotinia sclerotiorum. Mycologia 101:296-304.

Chadha, S., and Sharma, M. 2014. Transposable elements as stress adaptive capacitors induce genomic instability in fungal pathogen Magnaporthe oryzae. PLoS One 9:e94415.

Chen, F., Everhart, S. E., Bryson, P. K., Luo, C., Song, X., Liu, X., and Schnabel, G. 2015. Fungicide-induced transposon movement in Monilinia fructicola. Fungal Genet. Biol. 85:38-44.

Coleine, C., Masonjones, S., Sterflinger, K., Onofri, S., Selbmann, L., and Stajich, J. E. 2020. Peculiar genomic traits in the stress-adapted cryptoendolithic Antarctic fungus Friedmanniomyces endolithicus. Fungal Biol. 124:458-467.

Cubeta, M. A., Cody, B. R., Kohli, Y., and Kohn, L. M. 1997. Clonality in Sclerotinia sclerotiorum on infected cabbage in eastern North Carolina. Phytopathology 87:1000-1004.

Derbyshire, M., Denton-Giles, M., Hegedus, D., Seifbarghi, S., Rollins, J., van Kan, J., Seidl, M. F., Faino, L., Mbengue, M., Navaud, O., Raffaele, S., Hammond-Kosack, K., Heard, S., and Oliver, R. 2017. The complete genome sequence of the phytopathogenic fungus Sclerotinia sclerotiorum reveals insights into the genome architecture of broad host range pathogens. Genome Biol. Evol. 9:593-618.

dos Reis, T. F., Silva, L. P., de Castro, P. A., do Carmo, R. A., Marini, M. M., da Silveira, J. F., Ferreira, B. H., Rodrigues, F., Lind, A. L., Rokas, A., and Goldman, G. H. 2019. The Aspergillus fumigatus mismatch repair MSH2 homolog is important for virulence and azole resistance. MSphere 4: e00416-e00419.

Dowling, M. E., Bryson, P. K., Boatwright, H. G., Wilson, J. R., Fan, Z., Everhart, S. E., Brannen, P. M., and Schnabel, G. 2016. Effect of fungicide applications on Monilinia fructicola population diversity and transposon movement. Phytopathology 106:1504-1512.

Eisenman, H. C., Greer, E. M., and McGrail, C. W. 2020. The role of melanins in melanotic fungi for pathogenesis and environmental survival. Appl. Microbiol. Biotechnol. 104:4247-4257.

Fungicide Resistance Action Committee. 2012. FRAC list of plant pathogenic organisms resistant to disease control agents. https://www.frac.info/docs/ default-source/working-groups/sdhi-fungicides/group/list-of-resistantplant-pathogens_2012-edition.pdf

Galhardo, R. S., Hastings, P. J., and Rosenberg, S. M. 2007. Mutation as a stress response and the regulation of evolvability. Crit. Rev. Biochem. Mol. Biol. 42:399-435.

Gerstein, A.C., and Berman, J. 2020. Candida albicans genetic background influences mean and heterogeneity of drug responses and genome stability during evolution to fluconazole. bioRxiv 360347.

Gessler, N. N., Egorova, A. S., and Belozerskaya, T. A. 2014. Melanin pigments of fungi under extreme environmental conditions. Appl. Biochem. Microbiol. 50:105-113.

Gianessi, L. P., and Reigner, N. 2005. The value of fungicides in U.S. crop production. CropLife Foundation, Washington, DC. https://croplifefoundation.files.wordpress.com/2012/07/completed-fungicidereport.pdf

Gossen, B. D., Rimmer, S. R., and Holley, J. D. 2001. First report of resistance to benomyl fungicide in Sclerotinia sclerotiorum. Plant Dis. 85: 1206.

Gressel, J. 2011. Low pesticide rates may hasten the evolution of resistance by increasing mutation frequencies. Pest Manag. Sci. 67:253-257.

Harrison, B. D., Hashemi, J., Bibi, M., Pulver, R., Bavli, D., Nahmias, Y., Wellington, M., Sapiro, G., and Berman, J. 2014. A tetraploid intermediate precedes aneuploid formation in yeasts exposed to fluconazole. PLoS Biol. 12:e1001815.

Healey, K. R., Zhao, Y., Perez, W. B., Lockhart, S. R., Sobel, J. D., Farmakiotis, D., Kontoyiannis, D. P., Sanglard, D., Taj-Aldeen, S. J., 
Alexander, B. D., and Jimenez-Ortigosa, C. 2016. Prevalent mutator genotype identified in fungal pathogen Candida glabrata promotes multi-drug resistance. Nat. Commun. 7:11128.

Horváth, V., Merenciano, M., and González, J. 2017. Revisiting the relationship between transposable elements and the eukaryotic stress response. Trends Genet. 33:832-841.

Hwang, S., Kim, E., Lee, I., and Marcotte, E. M. 2015. Systematic comparison of variant calling pipelines using gold standard personal exome variants. Sci. Rep. 5:17875.

Jeon, J., Choi, J., Lee, G. W., Dean, R. A., and Lee, Y. H. 2013. Experimental evolution reveals genome-wide spectrum and dynamics of mutations in the rice blast fungus, Magnaporthe oryzae. PLoS One 8:e65416.

Kamvar, Z. N., Amaradasa, B. S., Jhala, R., McCoy, S., Steadman, J. R., and Everhart, S. E. 2017. Population structure and phenotypic variation of Sclerotinia sclerotiorum from dry bean (Phaseolus vulgaris) in the United States. PeerJ 5:e4152.

Kasuga, T., Bui, M., Bernhardt, E., Swiecki, T., Aram, K., Cano, L. M., Webber, J., Brasier, C., Press, C., Grünwald, N. J., and Rizzo, D. M. 2016. Host-induced aneuploidy and phenotypic diversification in the sudden oak death pathogen Phytophthora ramorum. BMC Genomics 17:385.

Keane, T. M., Wong, K., and Adams, D. J. 2013. RetroSeq: Transposable element discovery from next-generation sequencing data. Bioinformatics 29:389-390.

Kistler, H. C., and Miao, V. P. 1992. New modes of genetic change in filamentous fungi. Annu. Rev. Phytopathol. 30:131-153.

Knaus, B. J., and Grünwald, N. J. 2017. vcfR: A package to manipulate and visualize variant call format data in R. Mol. Ecol. Resour. 17:44-53.

Kohli, Y., and Kohn, L. M. 1998. Random association among alleles in clonal populations of Sclerotinia sclerotiorum. Fungal Genet. Biol. 23:139-149.

Koshiji, M., To, K. K. W., Hammer, S., Kumamoto, K., Harris, A. L., Modrich, P., and Huang, L. E. 2005. HIF-1 $\alpha$ induces genetic instability by transcriptionally downregulating MutS $\alpha$ expression. Mol. Cell 17:793-803.

Kuang, J., Hou, Y. P., Wang, J. X., and Zhou, M. G. 2011. Sensitivity of Sclerotinia sclerotiorum to fludioxonil: In vitro determination of baseline sensitivity and resistance risk. Crop Prot. 30:876-882.

Langmead, B., and Salzberg, S. L. 2012. Fast gapped-read alignment with Bowtie 2. Nat. Methods 9:357-359.

Lazarovits, G., Starratt, A. N., and Huang, H. C. 2000. The effect of tricyclazole and culture medium on production of the melanin precursor 1,8 dihydroxynaphthalene by Sclerotinia sclerotiorum isolate SS7. Pestic. Biochem. Physiol. 67:54-62.

Lehner, M. S., Paula Júnior, T. J., Silva, R. A., Vieira, R. F., Carneiro, J. E. S., Schnabel, G., and Mizubuti, E. S. G. 2015. Fungicide sensitivity of Sclerotinia sclerotiorum: A thorough assessment using discriminatory dose, $\mathrm{EC}_{50}$, highresolution melting analysis, and description of new point mutation associated with thiophanate-methyl resistance. Plant Dis. 99:1537-1543.

Li, H., Handsaker, B., Wysoker, A., Fennell, T., Ruan, J., Homer, N., Marth, G., Abecasis, G., and Durbin, R., and 1000 Genome Project Data Processing Subgroup. 2009. The sequence alignment map format and SAMtools. Bioinformatics 25:2078-2079.

Lucas, J. A., Hawkins, N. J., and Fraaije, B. A. 2015. The evolution of fungicide resistance. Adv. Appl. Microbiol. 90:29-92.

Ma, H. X., Chen, Y., Wang, J. X., Yu, W. Y., Tang, Z. H., Chen, C. J., and Zhou, M. G. 2009. Activity of carbendazim, dimethachlon, iprodione, procymidone and boscalid against Sclerotinia stem rot in Jiangsu Province of China. Phytoparasitica 37:421-429.

Maharjan, R. P., and Ferenci, T. 2017. A shifting mutational landscape in 6 nutritional states: Stress-induced mutagenesis as a series of distinct stress input-mutation output relationships. PLoS Biol. 15:e2001477.

Marçais, G., Delcher, A. L., Phillippy, A. M., Coston, R., Salzberg, S. L., and Zimin, A. 2018. MUMmer4: A fast and versatile genome alignment system. PLOS Comput. Biol. 14:e1005944.

Marchler-Bauer, A., and Bryant, S. H. 2004. CD-Search: Protein domain annotations on the fly. Nucleic Acids Res. 32(W):327-331.

Marchler-Bauer, A., Lu, S., Anderson, J. B., Chitsaz, F., Derbyshire, M. K., Deweese-Scott, C., Fong, J. H., Geer, L. Y., Geer, R. C., Gonzales, N. R., Gwadz, M., Hurwitz, D. I., Jackson, J. D., Ke, Z., Lanczycki, C. J., Lu, F., Marchler, G. H., Mullokandov, M., Omelchenko, M. V., Robertson, C. L., Song, J. S., Thanki, N., Yamashita, R. A., Zhang, D., Zhang, N., Zheng, C., and Bryant, S. H. 2011. CDD: A Conserved Domain Database for the functional annotation of proteins. Nucleic Acids Res. 39:D225-D229.

McDougall, P. 2016. The cost of new agrochemical product discovery, development and registration in 1995, 2000, 2005-8 and 2010 to 2014. R and D expenditure in 2014 and expectations for 2019. Consultancy study for CropLife International, CropLife America and the European Crop
Protection Association. https://croplife.org/wp-content/uploads/2014/04/ Phillips-McDougal-Research-and-Development-study.pdf

McKenna, A., Hanna, M., Banks, E., Sivachenko, A., Cibulskis, K., Kernytsky, A., Garimella, K., Altshuler, D., Gabriel, S., Daly, M., and DePristo, M. A. 2010. The Genome Analysis Toolkit: A MapReduce framework for analyzing next-generation DNA sequencing data. Genome Res. 20:1297-1303.

Miller, M. E., Zhang, Y., Omidvar, V., Sperschneider, J., Schwessinger, B., Raley, C., Palmer, J. M., Garnica, D., Upadhyaya, N., Rathjen, J., and Taylor, J. M. 2018. De novo assembly and phasing of dikaryotic genomes from two isolates of Puccinia coronata $\mathrm{f}$. sp. avenae, the causal agent of oat crown rust. MBio 9:e01650-17.

Mironenko, N. V., Alekhina, I. A., Zhdanova, N. N., and Bulat, S. A. 2000. Intraspecific variation in gamma-radiation resistance and genomic structure in the filamentous fungus Alternaria alternata: A case study of strains inhabiting Chernobyl reactor no. 4. Ecotoxicol. Environ. Saf. 45:177-187.

Molaei, H., Abrinbana, M., and Ghosta, Y. 2020. Baseline sensitivities to azoxystrobin and tebuconazole in Sclerotinia sclerotiorum isolates from sunflower in Iran related to sensitivities to carbendazim and iprodione. J. Phytopathol. 168:353-362.

Möller, M., Habig, M., Freitag, M., and Stukenbrock, E. H. 2018. Extraordinary genome instability and widespread chromosome rearrangements during vegetative growth. Genetics 210:517-529.

Moolhuijzen, P., See, P. T., Hane, J. K., Shi, G., Liu, Z., Oliver, R. P., and Moffat, C. S. 2018. Comparative genomics of the wheat fungal pathogen Pyrenophora tritici-repentis reveals chromosomal variations and genome plasticity. BMC Genomics 19:279.

O'Sullivan, D., Tosi, P., Creusot, F., Cooke, B. M., Phan, T. H., Dron, M., and Langin, T. 1998. Variation in genome organization of the plant pathogenic fungus Colletotrichum lindemuthianum. Curr. Genet. 33:291-298.

Otto-Hanson, L., Steadman, J. R., Higgins, R., and Eskridge, K. M. 2011. Variation in Sclerotinia sclerotiorum bean isolates from multisite resistance screening locations. Plant Dis. 95:1370-1377.

Parker, K. R., and von Borstel, R. C. 1987. Base-substitution and frameshift mutagenesis by sodium chloride and potassium chloride in Saccharomyces cerevisiae. Mutat. Res. 189:11-14.

Penaud, A., Huguet, B., Wilson, V., and Leroux, P. 2003. Fungicide resistance of Sclerotinia sclerotiorum in French oilseed rape crops. Pages 1097-1098 in: Proceedings of 11 th International Rapeseed Congress. Copenhagen, Denmark.

R Core Team. 2019. R: A language and environment for statistical computing. $\mathrm{R}$ Foundation for Statistical Computing, Vienna, Austria. https://www.Rproject.org

Schnabel, G., Chen, F., Everhart, S. E., Bridges, W. C., and Liu, X. 2014. Studies on sensitivity reduction in solo and mixture treatments and fungicide-induced mutagenesis in Monilinia fructicola. Modern fungicides and antifungal compounds VII: Proceedings of the 17th International Reinhardsbrunn Symposium, Friedrichroda, Germany.

Shapiro, R. S. 2015. Antimicrobial-induced DNA damage and genomic instability in microbial pathogens. PLoS Pathog 11:e1004678.

Shor, E., Fox, C. A., and Broach, J. R. 2013. The yeast environmental stress response regulates mutagenesis induced by proteotoxic stress. PLoS Genet. 9:e1003680.

Shor, E., and Perlin, D. S. 2015. Coping with stress and the emergence of multidrug resistance in fungi. PLoS Pathog 11:e1004668.

Terán, H., Lema, M., Schwartz, H. F., Duncan, R., Gilbertson, R., and Singh, S. P. 2006. Modified Petzoldt and Dickson scale for white mold rating of common bean. Annu. Rep. Bean Improv. Coop. 49:115.

Tóthová, M., Hudec, K., and Tóth, P. 2019. Sensitivity of Sclerotinia sclerotiorum to strobilurin fungicides in Slovakia. Plant Prot. Sci. 56:13-17.

Treangen, T. J., and Salzberg, S. L. 2012. Repetitive DNA and next-generation sequencing: computational challenges and solutions. Nat. Rev. Genet. 13: 36-46.

Troncoso-Rojas, R., Báez-Flores, M. E., Pryor, B., García, H. S., and Tiznado-Hernández, M. E. 2013. Inter simple sequence repeat polymorphism in Alternaria genomic DNA exposed to lethal concentrations of isothiocyanates. Afr. J. Microbiol. Res. 7:838-852.

van den Bosch, F., Paveley, N., Shaw, M., Hobbelen, P., and Oliver, R. 2011. The dose rate debate: Does the risk of fungicide resistance increase or decrease with dose? Plant Pathol. 60:597-606.

Wickham, H., François, R., Henry, L., and Müller, K. 2020. dplyr: A grammar of data manipulation. $\mathrm{R}$ package version 0.8.5. https://cran.r-project.org/ web/packages/dplyr/index.html

Wickham, H., and Henry, L. 2020. tidyr: Tidy messy data. R package version 1.1.0. https://cran.r-project.org/web/packages/tidyr 